\title{
An Extension of Scalable MAC for VANETs
}

\author{
Ganis Zulfa Santoso and Monsoo Kang
}

\begin{abstract}
Vehicular ad hoc networks (VANETs) are networks of vehicles either to directly communicate with each other or to have access to the Internet with base stations (BSs) along roads. As the number of cars is increasing every year, designing a scalable MAC to provide VANETs is getting more crucial. In this paper, we proposed an extended scalable MAC protocol. Our simulation results show the scalability of our protocols as it performs exceptionally well in very high density networks.
\end{abstract}

Index Terms - Contention window, MAC, scalable, VANETs.

\section{INTRODUCTION}

Vehicular ad hoc networks (VANETs) are networks of vehicles either to directly communicate with each other or to have access to the Internet with base stations (BSs) along roads. The networks will be an infrastructure of the Intelligent Transportation System (ITS). Especially for safety on the road, VANETs is going to take an important role, which enables a broadcast on the current traffic information. The information will be carried in a broadcast packet to surroundings.

As the number of cars is increasing every year [1], designing a scalable MAC protocol to provide VANETs with sustainability is getting more crucial. One of the distinguished attributes of VANETs is its high dynamic movement of the nodes, thereby car density sometimes becomes so sparse that data collisions rarely happen or car traffic becomes so congested that the networks will severely experience data collisions.

In this paper, we have extended VMAC [2] by adding three additional modifications such as mutual BEB algorithm, broadcast priority, and reset backoff algorithm, which make VMAC more resilient for heavily congested VANETs.

The rest of the paper is organized as follows. In Section II we introduce the related work. In the next section, VMAC and the modifications are explained in more details. The extensive simulations and the results are stated in Section IV. The last section will finalize the paper with the conclusions and future work.

\section{RELATED WORK}

This work based on the scalable MAC introduced in [2] is proposing three modifications. The first is the modification of backoff algorithm for which numerous ideas already were proposed in the literature [3]-[5]. But the ideas were not fully

Manuscript received October 15, 2012; revised November 18, 2012.

The authors are with the Department of Computer Engineering, Chosun University, Gwangju, South Korea (e-mail: mskang@chosun.ac.kr; ganiszulfa@gmail.com ) tailored to be adapted for dynamic and fast changes in VANETs. The work in [7-9] is adjusting the size of content window to perform a successful broadcast. [10] and [11] try to accomplish it by extending RTS/CTS scheme.

This paper proposes a few of novel ideas to implement a scalable MAC for VANETs. The purpose of the protocol is to be a base to develop safety applications of VANETs by achieving more successful broadcast as well as satisfying high throughput for another applications e.g., video and audio.

\section{EXTENDED SCALABLE MAC (VMAC EXT)}

The purpose of the proposed MAC is to sustain the performance of VANET even with the huge number of transmission competitions. It is already well known that higher network density causes the CSMA/CA [12] based wireless networks such as IEEE $802.11 \mathrm{a}$, b, and p more unstable [13]. Thus the original VMAC is proposing to combine TDMA with CSMA to take advantage of each feature in the sense that a heavily congested network prefers TDMA to CSMA and vice versa CSMA is better than TDMA in a sparse network. To achieve higher sustainability, we have extended the VMAC [2] by adding three additional features.

\section{A. $V M A C$}

The performance of VMAC results from mitigating contentions per unit time by assigning different priorities to different nodes in temporal order. The VMAC employs a number of timeslots corresponding to TDMA. Each node with VMAC may select more than one timeslot during which the node will be the owner of the slots and gain a higher priority to transmit data with smaller contention window similar to the IEEE $802.11 \mathrm{e}$ [15]. The other nodes not owning the slots will also choose but bigger contention window to have a little chance to transmit data. For more details, please refer to [2]. The number of selected slots owned by a node depends on the degree of network congestion.

\section{B. Mutual BEB Algorithm (MBEB)}

When a VANET becomes severely congested, which means the network has a large number of transmission competitions in a small area, in order to avoid simultaneous transmissions or collisions, the range of contention window should definitely become large. The IEEE 802.11 accommodates this by applying the BEB algorithm. If a collision happens due to the simultaneous transmissions of two or more nodes, the collided nodes will double their CWs up and select each CW again. If collisions happen repeatedly, the range of each $\mathrm{CW}$ will be exponentially increased again and again. Eventually, the size of $\mathrm{CW}$ will be big enough to avoid the next collision. 
In the normal BEB algorithm, the increased $\mathrm{CW}$ returns to the minimum $\mathrm{CW}$ after a successful transmission. This reset seems to be suboptimal since the number of competing nodes in a highly saturated VANET is not likely to be suddenly reduced and another of collisions may be triggered. However, it will be a problem that the collision experienced nodes are keeping the increased $\mathrm{CW}$ while the nodes not experiencing collisions yet, relatively maintain a smaller $\mathrm{CW}$. Because the nodes with smaller CWs may have more opportunity to transmit data, the unfairness in packet transmission comes about.

We call our modification of BEB mutual BEB. We assume the nodes not directly involving a transmission can recognize if the transmission is successfully complete or not by overhearing the data packet and the ACK exchanged by the transmission peers. Thus, every time a node either directly detects or overhears a collision, it will double up its own CW. This operation will increase the CWs of all neighbor nodes together around the collision. In addition to the collision, every time a node either successfully transmits a packet or overhears the successful transmission, it does not change its $\mathrm{CW}$ to the minimum $\mathrm{CW}$. but the $\mathrm{CW}$ is divided by two as follows:

$$
\begin{gathered}
C W=\min \left[2 \times C W, C W_{\max }\right] \text { upon a collision, } \\
C W=\max \left[C W / 2, C W_{\min }\right] \text { upon a success. }
\end{gathered}
$$

This is assuring the VMAC of the fairness among all nodes as much as possible and reducing the probability of collision. The MILD algorithm [5] can be used instead of MBEB. However, the MILD algorithm is not much adaptive to high dynamics of VANETs since the $\mathrm{CW}$ decrease is linear. Fig. 1 shows the mechanism of MBEB.

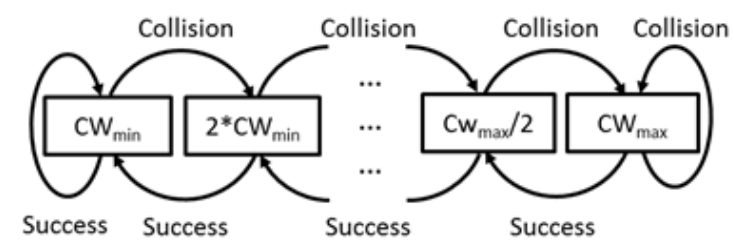

Fig. 1. Backoff mechanism of MBEB. nodes will change the CW for its own collision/overhearing the collision or a successful transmission/ overhearing an ACK.

\section{Reset Backoff Algorithm (RBA)}

In the IEEE 802.11 DCF, a node freezes its backoff once it detects the medium becomes busy because of someone's transmission and continue to count the backoff again when the medium becomes free after the transmission. We believe that this operation is not beneficial to VANET especially when its network density is frequently changed.

The backoff mechanism is introduced so that the nodes, which want to access the medium at a time, can avoid the simultaneous transmission or collision. Assuming the nodes selected the backoff time at the same time, if the backoff times randomly selected by the nodes are different, the times forces the start time of each node's transmission to be different. The backoff freezing during other's transmission is introduced for the nodes earlier triggering the backoffs to keep a kind of priority to the nodes later triggering the backoffs. In other words, if a transmission is finished and the current frozen backoffs resume their countdown, their remaining times will be smaller than the newly started backoffs probabilistically. Thus, the transmissions of the former nodes would like to start earlier than the latter nodes probabilistically. However, The backoff freezing is harmful for VMAC because the freezing interrupts the operations of VMAC. VMAC gives a higher priority to the node owning a timeslot by giving smaller $\mathrm{CW}$ to the owner node during the timeslot. If we permit the backoff freezing in VMAC, the non-owner nodes with lower priority during the time slot will gain the chances to transmit data with similar frequency to the owner nodes even during the time slot. Instead of using the backoff freezing, furthermore, VMAC does reset all the current backoffs after a transmission to mitigate or scatter out contentions over time. If every node selects its $\mathrm{CW}$ again except the one doing the previous transmission, the effective and real competition occurs only among the owner nodes because the owners and the non-owners have the different ranges of $\mathrm{CW}$ during the timeslot, which results in scattering out the contentions over time. In other words, VMAC tries to limit the number of competitors by allowing only a small number of owners to repeatedly send data during the timeslot. If we do not reset the backoffs after every transmission, some of the following non-owner nodes would like to have the next transmission due to the effect of freezing at the same backoff expiry time with some of other owner nodes. To eliminate the situation as much as possible, we are proposing to use backoff resetting which we call RBA.

Fig. 2 shows an example how to apply the RBA among owner nodes. For the conventional backoff algorithm, the remaining backoff time will be used in the next round. But in the RBA, the remaining backoff time will be discarded and each node will choose a new backoff time in every transmission.

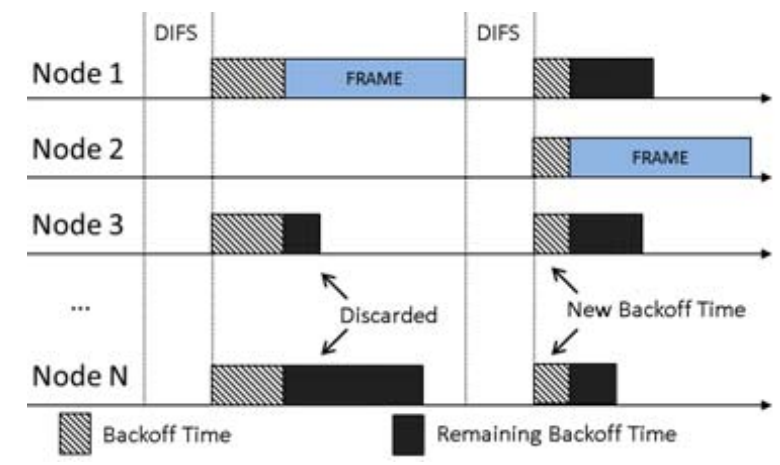

Fig. 2. the Example of reset Backoff algorithm (RBA).

If we look at Fig. 2, Node 2 initially doesn't have any packet to transmit during the first transmission. The other owner nodes selected their backoffs uniformly distributed on the range of $\mathrm{CW}$. Assuming we use the original backoff, the other owner nodes during Node 1's transmission might keep their backoff and Node 2 will independently choose its own backoff time. Since other owner nodes have already started counting their backoff times down, Node 2 will not have a fair chance to compete. For fairness, all the owner nodes except the first transmitter will select their CWs again.

Some may argue that because the previous backoff time is discarded, it will not be fair for those who have kept it. But for one node to consecutively get the least backoff time will 
be very unlikely and vice versa, especially when the number of nodes is really high.

\section{Broadcast Priority Algorithm (BPA)}

A node having a broadcast packet has a very small backoff range. The small backoff range assures the broadcast packets to have a higher priority than other packets. In order to take the network density into account, the backoff value should be calculated by a function of the current value of $\mathrm{CW}$. The following explains the algorithm in details:

backoff $=\operatorname{rand}[0, L \times C W / C W m i n]$ for broadcast packets, backoff $=\operatorname{rand}[0, C W]+L \times C W / C W m i n$ for other packets.

$L$ is the leverage variable for broadcast packet. The value of $L$ could be any positive integer. Applying the algorithm gives broadcast packets a higher priority than other packets. However, collisions still may exist among broadcast packets. The higher the value of $L$, the probability of collision between broadcast packets is lower and vice versa. However, if the value of $L$ is too big it will create a high delay for other packets beside broadcast packets. Thus, determining a good value of $L$ will be very important.

\section{Simulation Results}

The extensive simulations have been performed with NS-3.13 on Ubuntu 11.04. Each simulation is repeated 10 times with different random seeds to observe an averaged behavior. All nodes are located within a single hop distance to annihilate the effects of routing protocol. Because the initial part of the simulation data is including the transient response of the network, we have considered and averaged only the simulation data of the second half $(10 \mathrm{sec}$. $)$ out of a single simulation runtime $(20 \mathrm{sec}$.).

Every sender generates $400 \mathrm{Kbps}$ whereas capacity of the wireless link is set to $12 \mathrm{Mbps}$. We are using $802.11 \mathrm{p}$ where the $C \mathrm{Wmin}=16$ and $C \mathrm{Wmax}=512$. The size of each transmission packet is set to 512 bytes. The broadcast packets are sent randomly every two seconds with the size of each of them is 8 bytes. In simulation we compare the original 802.11p, original VMAC, and the extended VMAC. For both VMAC, the number of slot is fixed to 4 and each sender own a single slot. In BPA for VMAC Ext, the $L$ value that we use is 4 . The parameters that we measured are broadcast delivery ratio, data throughput and collisions rate.

\section{A. Broadcast Delivery}

In a safety application, broadcast packets are important in term of gathering the information about the surrounding of a vehicle. As a safety application [14] can save human life on the road, it became our main goal in our research. The rising of entertainment applications in a way is hindering the deliverance of broadcast packets as they are competing in the same medium. Therefore we add the broadcast priority feature in our protocol as explained in the previous section.

Fig. 3 shows that our protocol has a successful delivery rate of more than $93 \%$ regardless how dense the network is. This provides the reliability and robustness for safety applications in VANETs.
The delivery of the broadcast packets may not be $100 \%$, it doesn't mean that we are neglecting the information of the undelivered packets to jeopardize the safety on the road. We can create a system where the information of the delivered packets covers the undelivered one. However, if the performance is not satisfying, we can always increase the value of $L$ in BPA.

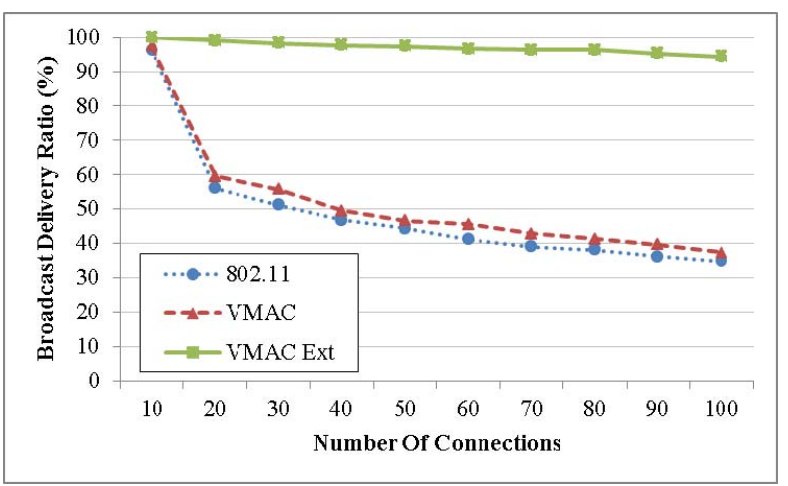

Fig. 3. Broadcast packet delivery rate of VMAC ext compared to another protocols.

\section{B. Channel Utilization}

Even though broadcast delivery is our main objective in this research, our protocol also performed stunningly in data throughput. As shown by the Figure 4, even when the performance of other protocols is waning, our protocol is still pushing the limit of medium capacity.

The improvements of VMAC Ext protocol because the MBEB algorithm is increasing $\mathrm{CW}$ of all nodes fast. And also, RBA and VMAC are working together to mitigate the contention. BPA is also aiding by isolating the broadcast packets to direct transmission packets. This combination is tremendously beneficial in reducing the collisions as also shown in Fig. 5. Our protocol successfully reduces the collision rate to the minimum therefore improving the throughput data significantly.

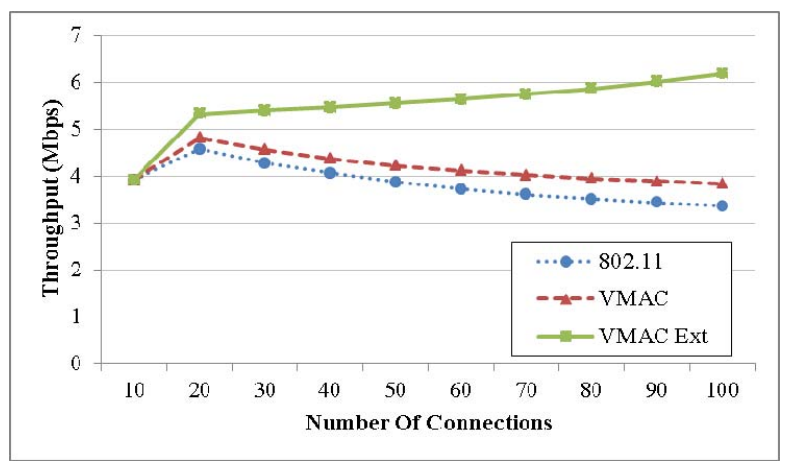

Fig. 4. Data throughput of VMAC ext compared to another protocols.

Looking at Fig. 4, readers can be riddled on how after 20 connections, data throughput of VMAC Ext seems to be increasing instead of decreasing like other protocols. Keep in mind that at Fig. 5, the total throughput is also calculating the broadcast packet delivery in each node. For better view on how VMAC Ext increases channel utilization, please refer to Fig. 6 where we ignore the broadcast packet in calculation. From the figure we can see at 10 connections, all protocols can perform well and can transmit all $400 \mathrm{Kbps}$ with few problems. But by the increase of the connections, VMAC Ext improves the performance of other protocols even more. 


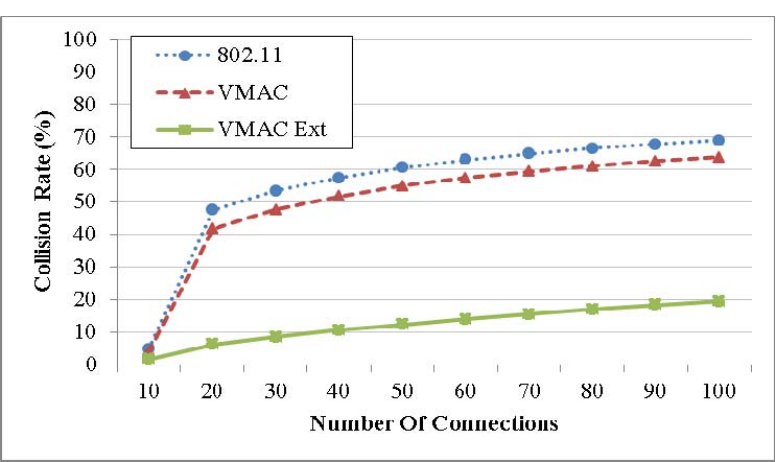

Fig. 5. Collision rate of VMAC ext compared to another protocols.

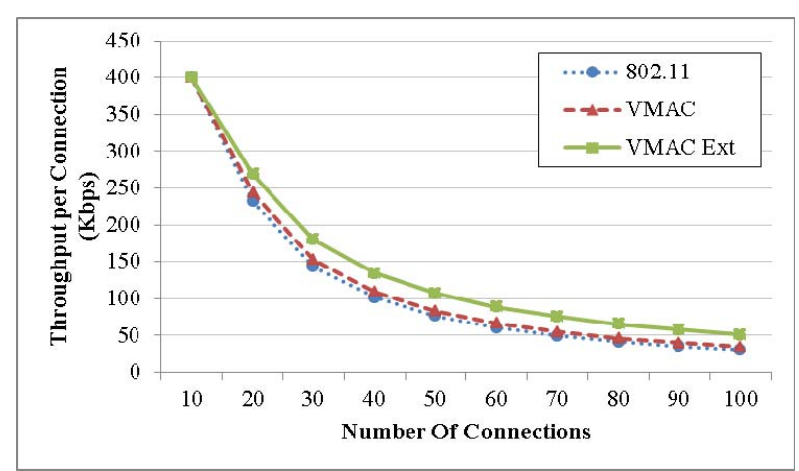

Fig. 6. Throughput per connections comparisons.

\section{CONCLUSION}

The IEEE 802.11 is not optimum for an area with a high number of competing nodes as it performs poorly. By the unavoidable increasing number of vehicles in the world, it is a necessary for a MAC to be scalable. Therefore in this paper we have presented the algorithm of our protocol and the simulation result to prove its scalability. Our protocol performed superior even in a very high density network proving that it is a very scalable and reliable MAC.

We have explained each algorithm elaborately on how it is beneficial for high density network. And our study shows that the addition of BPA, MBEB, and BPA into the VMAC is increasing the VMAC performance significantly by providing a better channel utilization and reducing collisions.

For the foreseeable future, there are two main applications of VANETs; safety and entertainment applications. For both applications, VMAC Ext proven to be very efficient as it can perform exceptionally well in broadcast delivery ratio and also data throughput.

The algorithm in finding the optimum number of slots in VMAC is remained as future work. It depends also on the capability of measuring the network density. The optimum value of $L$ in BPA algorithm should also be considered as future work even though when it was set as 4 it performed really well in this simulation. The mobility of the nodes should also be considered for the future works as the number of nodes will not always be fixed especially in VANETs environment where number of nodes are dynamically change. Our work focused on the transmission within one single hop, extensive researches for multiple hops should also be considered for the future works.

\section{ACKNOWLEDGMENT}

This work is supported by the IT R\&D program of KCC/KCA (11911-05003: R\&D on Smart Node Technology for Cloud Networking and Contents Centric Networking).

\section{REFERENCES}

[1] Number of U. S. Aircraft. [Online]. Available: http://www.bts.gov/publications/national_transportation_statistics/ht $\mathrm{ml} /$ table 01 11.html

[2] K. Moonsoo et al., "A scalable MAC for vehicular Ad hoc networks," Networks and Optical Communications (NOC), 2011.

[3] C. R. Krishna, S. Chakrabarti, and D. Datta, "A Modified Backoff Algorithm for IEEE 802.11 DCF- Based MAC Protocol In A Mobile Ad Hoc Network," in Proc. of IEEE Region 10 Conference, pp. 664 667, vol. 2, November 2004.

[4] J. Deng, P. K. Varshney, and Z. J. Haas, "A New Backoff Algorithm for the IEEE 802.11 Distributed Coordination Function," Networks and Optical Communications (NOC), pp. 204-207, July 2011.

[5] N. O. Song, B. Kwak, J. Song, and L. E. Miller, "Enhancement of IEEE 802.1 1 Distributed Coordination Function with Exponential Increase Exponential Decrease Backoff Algorithm," Vehicular Technology Conference, pp. 2775 - 2778, April 2003.

[6] I. Elarbaoui and H. H. Refai, "Enhancement of IEEE 802.11 DCF Backoff Algorithm under Heavy Traffic," Networks and Optical Communications (NOC), pp. 204-207, July 2011.

[7] S. W. Chang, J. Cha, and S. S. Lee, "Adaptive EDCA Mechanism for Vehicular Ad-hoc Network," in Proc. of 2012 International Conference on Information Networking (ICOIN), Feb. 2012.

[8] R. Stanica, E. Chaput, and A. L. Beylot, "Broadcast Communication in Vehicular Ad-Hoc Network Safety Applications," in Proc. of 2011 IEEE Consumer Communications and Networking Conference (CCNC), 2011.

[9] F. Ye, R. Yim, J. Guo, J. Zhang, and S. Roy, "Prioritized Broadcast Contention Control in VANET," in Proc. of Communications (ICC), 2010 IEEE International Conference, pp. 1- 5, May 2010.

[10] K. Xu, M. Gerla, and S. Bae, "How Effective is the IEEE 802.11 RTSKTS Handshake in Ad Hoc Networks," 2002.

[11] A. T. Toyserkani, E. G. Strom, and A. Svensson, "An Efficient Broadcast MAC Scheme for Traffic Safety Applications in Automotive Networks," in Proc. of IEEE Wireless Communications and Networking Conference, April 2006.

[12] A. S. Tanenbaum, Computer Networks, Prentice Hall PTR, 2003.

[13] B. Giuseppe, "Performance analysis of the IEEE 802.11 distributed coordination function," in Proc. of Selected Areas in Communications, IEEE Journal on 18, no. 3, pp. 535-547, 2000

[14] G. Z. Santoso and M. Kang, "Performance Analysis of AODV, DSDV and OLSR in a VANETs Safety Application Scenario," in Proc. of 2012 14th International Conference on Advanced Communication Technology (ICACT), pp. 57 - 60, May 2010.

[15] G. Antonio and M. Nunes. "Performance evaluation of IEEE 802.11 e," in Personal, Indoor and Mobile Radio Communications the 13th IEEE International Symposium on, vol. 1, pp. 511-517. 2002.

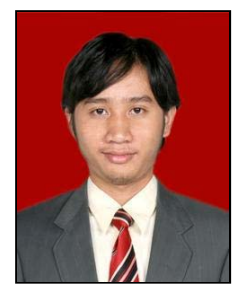

Ganis Zulfa Santoso received the B.Eng degree in Electrical Engineering from University of Indonesia, Jakarta, Indonesia, in 2008. From 2009 to 2011, he worked at PT SML Technologies, Jakarta, Indonesia. In 2011, he was selected as Global IT Scholarship grantee. Currently, he is a graduate student in the Dept. of Computer Engineering, Chosun University, South Korea. His current research interests are network protocols on vehicular ad hoc networks.

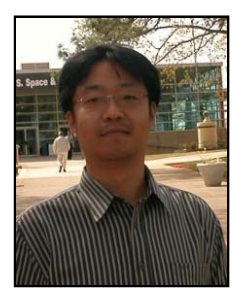

Moonsoo Kang received the B.S degree in Computer Science from Korea Advanced Institute of Science and Technology (KAIST), Daejon, Korea, in 1998. He received the M.S. and Ph.D. degrees in Engineering from Information and Communications University (ICU), Daejon, Korea, in 2000 and in 2007 respectively, which is currently a part of KAIST. Since $2007, \mathrm{He}$ is an assistant professor with the School of Computer Engineering, Chosun University in Gwangju, Korea. His research interests are network protocols on ad hoc networks such as sensor, mesh and vehicular networks. 